in vivo $34: 1921-1929(2020)$

doi:10.21873/invivo.11989

\title{
Glasgow Prognostic Score (GPS) and Tumor Response as Biomarkers of Nivolumab Monotherapy in Third- or Later-line Setting for Advanced Gastric Cancer
}

\author{
TAKASHI KUROSAKI ${ }^{1}$, HISATO KAWAKAMI ${ }^{1}$, SEIICHIRO MITANI ${ }^{1}$, RYOHEI KAWABATA ${ }^{2}$, \\ TAKAYUKI TAKAHAMA ${ }^{3}$, YOSHIKANE NONAGASE ${ }^{4}$, SOICHI FUMITA ${ }^{1}$, TOMOHIRO OZAKI ${ }^{4}$, \\ YASUTAKA CHIBA $^{5}$, TAKAO TAMURA ${ }^{3}$ and KAZUHIKO NAKAGAWA ${ }^{1}$ \\ ${ }^{l}$ Department of Medical Oncology, Kindai University Faculty of Medicine, Osaka-Sayama, Japan; \\ ${ }^{2}$ Department of Surgery, Osaka Rosai Hospital, Sakai, Japan; \\ ${ }^{3}$ Department of Medical Oncology, Kindai University Nara Hospital, Ikoma, Japan; \\ ${ }^{4}$ Department of Medical Oncology, Kishiwada City Hospital, Kishiwada, Japan; \\ ${ }^{5}$ Clinical Research Center, Kindai University Hospital, Osaka-Sayama, Japan
}

\begin{abstract}
Background/Aim: This study aimed to seek clinical biomarkers of nivolumab monotherapy for advanced gastric cancer (AGC) of which efficacy is limited. We focused on Glasgow Prognostic Score (GPS), which reflects systemic inflammatory and nutritional status as well as disease control by chemotherapy immediately before nivolumab (DCBC). Patients and Methods: AGC patients with measurable lesions who were treated with nivolumab in the third-or later-line were included. DCBC was defined as a best overall response of complete response (CR), partial response, stable disease, or non-CR/non-progressive disease achieved by chemotherapy immediately before nivolumab. Results: Eighty patients were analyzed. Among the various clinical factors, multivariable analysis revealed that a GPS of 2 was significantly associated with a shorter overall survival and DCBC was significantly associated with a longer progression-free survival. Conclusion: We present the potential of GPS and DCBC as efficient biomarkers of nivolumab for AGC, that warrants further evaluation.
\end{abstract}

Immune checkpoint inhibitors (ICIs) have recently been found to confer clinical benefit in patients with various types

This article is freely accessible online.

Correspondence to: Hisato Kawakami, MD, Ph.D., Department of Medical Oncology, Kindai University Faculty of Medicine, 377-2 Ohno-higashi, Osaka-sayama, Osaka 589-8511, Japan. Tel: +81723660221, Fax: +81723605000, e-mail: kawakami_h@ med.kindai.ac.jp

Key Words: Advanced gastric cancer, immune checkpoint inhibitors, nivolumab, Glasgow Prognostic Score (GPS), biomarkers. of advanced solid tumors (1-4). Nivolumab, a fully human monoclonal immunoglobulin G4 against programmed cell death-1 (PD-1), is one of the most widely administered ICIs and shows efficacy for several types of cancers, contributing to durable survival in patients in the palliative setting. With regard to advanced gastric cancer (AGC), nivolumab monotherapy conferred a survival benefit in heavily pretreated patients in a global phase III trial (5), although the proportion of patients who showed such benefit was smaller compared to other types of solid tumors, with a response rate of only $11 \%$, highlighting the importance of identification of individuals likely to benefit from this treatment.

Attempts to develop biomarkers for ICI treatment of AGC have thus far identified only high microsatellite instability (MSI-H) (6) and tumor expression of the PD-1 ligand PDL1 (7). Although other candidate biomarkers such as Epstein-Barr virus positivity and PIK3CA mutation have been proposed $(8,9)$, the value of such information is uncertain and patients accessible to these examinations are limited.

The Glasgow prognostic score (GPS) is an objective scoring tool that is based on the serum concentrations of $\mathrm{C}$ reactive protein (CRP) and albumin and thus reflects the systemic inflammatory and nutritional status (10). GPS has been identified as a prognostic factor for various types of cancers (11-13) and as a predictive factor for adjuvant chemotherapy in gastric cancer patients after curative surgery (14), for which the immune response has been implicated as a key determinant. No data are available with regard to the predictive or prognostic value of GPS for nivolumab treatment in patients with AGC.

Short-term induction with certain chemotherapeutic agents was recently shown to enhance sensitivity to subsequent ICI 


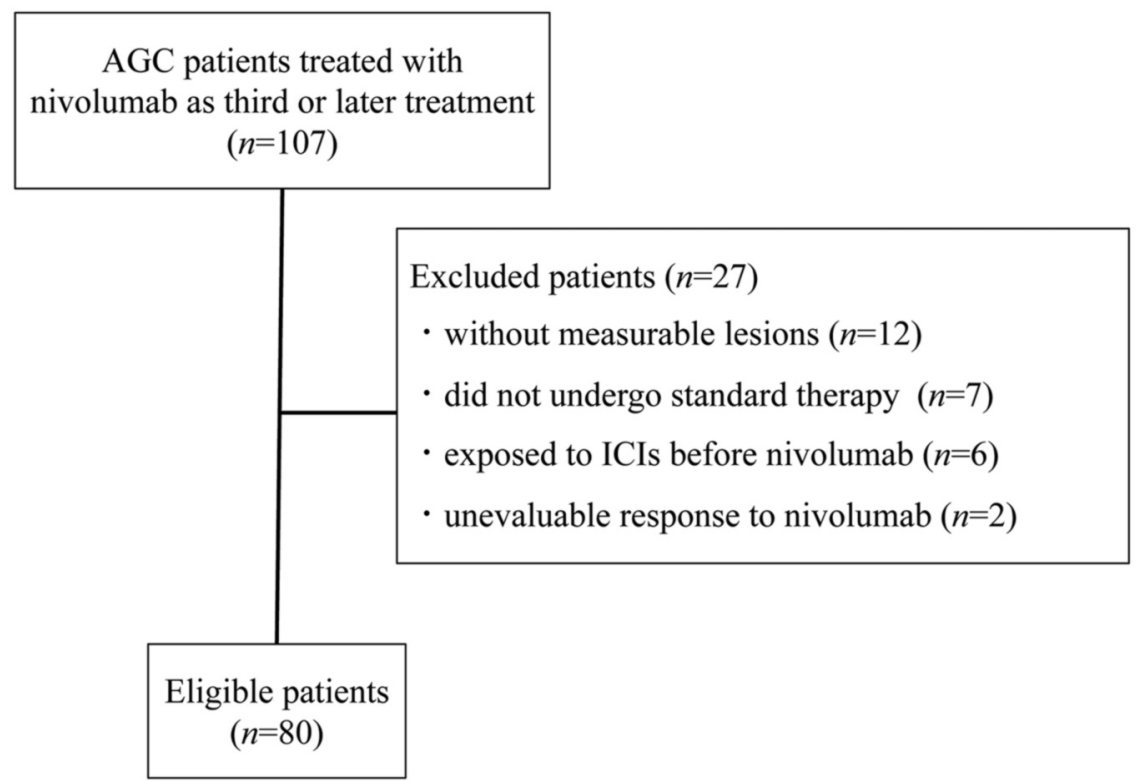

Figure 1. Flow diagram of the study. ICIs, Immune checkpoint inhibitors; AGC, advanced gastric cancer.

treatment in metastatic triple-negative breast cancer (15). Besides, achievement of a clinical response to chemotherapy immediately before nivolumab was shown to increase the likelihood of disease control obtained by nivolumab in nonsmall cell lung cancer (16), of which concept may be applied to AGC. We have, therefore, herein assessed a large group of unselected, consecutive AGC patients who were treated with nivolumab in the clinical practice setting in an attempt to identify potential biomarkers of nivolumab for AGC among the various clinical factors including GPS and the response to chemotherapy administered immediately prior to nivolumab.

\section{Patients and Methods}

Patients. We retrospectively reviewed the medical records of all patients with unresectable advanced or recurrent gastric or gastroesophageal junction cancer who were treated with nivolumab monotherapy at the Kindai University Hospital, Kindai University Nara Hospital, Kishiwada City Hospital, or Osaka Rosai Hospital between October 2017 and March 2019. Patients who met the following eligibility criteria before nivolumab therapy were enrolled in the study: a history of standard therapy with two or more regimens including pyrimidine analogs and taxanes, an Eastern Cooperative Oncology Group (ECOG) performance status (PS) of 0 to 2 , adequate organ function, no prior ICI treatment, and the presence of a measurable lesion. We excluded patients whose response to nivolumab was unevaluable. Nivolumab was administered intravenously at a dose of $3 \mathrm{mg} / \mathrm{kg}$ or $240 \mathrm{mg} /$ body every 2 weeks. The study protocol was approved by the institutional review board of each participating hospital (Kindai University Hospital, No. 29-115).
Data collection. We collected information on the following clinicopathological features: sex, age, ECOG PS, histological type, HER2 status, number of organs with metastases, presence of liver metastasis, serum levels of lactate dehydrogenase (LDH), CRP, and albumin, and white blood cell differential at the time of the first nivolumab administration. We also retrieved treatment history and tumor response to the chemotherapy administered immediately before the onset of nivolumab treatment. Tumor response was assessed according to the Response Evaluation Criteria in Solid Tumors version 1.1 (RECIST1.1). We defined overall response rate (ORR) as the proportion of patients with a best overall response of complete response (CR) or partial response (PR), and disease control rate (DCR) as the proportion of patients with a best overall response of CR, PR, or stable disease (SD). Progression-free survival (PFS) was measured from the time of treatment initiation to clinical or radiographic progression or death from any cause. Overall survival (OS) was measured from the time of treatment initiation to death from any cause. Patients without documented clinical or radiographic disease progression or who were still alive were censored at the last follow-up.

We recorded GPS at the time of first administration of nivolumab. Patients with an elevated CRP level $(>1.0 \mathrm{mg} / \mathrm{dl})$ and hypoalbuminemia $(<3.5 \mathrm{~g} / \mathrm{dl})$ were assigned a score of 2 . Those with only one of these abnormalities were assigned a score of 1 , and those with neither a score of 0 . The neutrophil/lymphocyte ratio (NLR) at the time of the first administration of nivolumab was also recorded, and we specified 5 as the cutoff between a high and low NLR as in previous studies $(17,18)$.

Statistical analysis. PFS and OS were compared according to baseline characteristics with the use of a Cox proportional hazards model, with the hazard ratio (HR) and its $95 \%$ confidence interval (CI) being presented. A $p$-value of $<0.2$ in univariable analysis was selected as explanatory variable for multivariable analysis. Survival 
Table I. Patient characteristics.

\begin{tabular}{lcc}
\hline Characteristic & No. of patients & $\%$ \\
\hline $\begin{array}{l}\text { Median age (range), years } \\
\text { Gender, male/female }\end{array}$ & \multicolumn{2}{c}{$71(43-87)$} \\
ECOG PS, 0/1/2 & $14 / 54 / 12$ & $17.5 / 67.5 / 15.0$ \\
Histology, differentiated/ & $49 / 22 / 9$ & $61.3 / 27.5 / 11.3$ \\
$\quad$ undifferentiated/unknown & & \\
HER2 status, positive/negative & $16 / 64$ & $20 / 80$ \\
No. of metastatic sites, $1 / \geq 2$ & $26 / 54$ & $32.5 / 67.5$ \\
Liver metastasis, yes/no & $36 / 44$ & $45 / 55$ \\
Previous gastrectomy, yes/no & $40 / 40$ & $50 / 50$ \\
No. of previous regimens, & $50 / 18 / 12$ & $62.5 / 22.5 / 15.0$ \\
2/3/ $\geq 4$ & & $78.8 / 21.3$ \\
Neutrophil/lymphocyte ratio, & $63 / 17$ & $37.5 / 40.0 / 22.5$ \\
$\quad<5 / \geq 5$ & & $0 / 25.0 / 47.5 / 21.3 / 6.3$ \\
GPS, 0/1/2 & $30 / 32 / 18$ & \\
$\begin{array}{l}\text { Best overall response to } \\
\text { chemotherapy before }\end{array}$ & $0 / 20 / 38 / 17 / 5$ & \\
nivolumab, CR/PR/SD/PD/NA & & \\
\hline
\end{tabular}

ECOG PS, Eastern Cooperative Oncology Group performance status; HER2, human epidermal growth factor receptor 2; GPS, Glasgow prognostic score; CR, complete response; PR, partial response; SD, stable disease; PD, progressive disease; NA, not available.

was also analyzed with the Kaplan-Meier method and was compared between groups with the log-rank test. Comparison of ORR or DCR according to baseline characteristics was performed with the Fisher's exact test. All statistical analyses were performed with EZR (Saitama Medical Center, Jichi Medical University, Saitama, Japan), a graphical user interface for R (The R Foundation for Statistical Computing, Vienna, Austria). A $p$-value of $<0.05$ was considered statistically significant.

\section{Results}

Patient characteristics. A search of medical records at four Institutions identified 107 patients who were treated with nivolumab for AGC. Of these patients, 80 individuals met the eligibility criteria and were enrolled in the present study (Figure 1); their clinical characteristics are summarized in Table I. All 80 patients received nivolumab as a third- or laterline treatment. The median age at the onset of nivolumab treatment was 71 years (range $=43-87$ years). Thirty $(37.5 \%)$, $32(40.0 \%)$, and $18(22.5 \%)$ patients had a GPS of 0,1 , and 2 , respectively. The median serum LDH level was 234 IU/1 (range $=114-1077$ IU/1). The most commonly administered chemotherapy regimen immediately before the onset of nivolumab treatment was ramucirumab plus paclitaxel (48.8\%), followed by irinotecan monotherapy (17.5\%), paclitaxel monotherapy (11.3\%), nanoparticle albumin-bound paclitaxel monotherapy (6.3\%), and others (16.3\%). The best overall response to the chemotherapy administered immediately before nivolumab treatment was evaluable in 75
(93.8\%) of the 80 patients. We defined disease control by the chemotherapy administered immediately prior to nivolumab treatment (DCBC) as a best overall response to such chemotherapy of $\mathrm{CR}, \mathrm{PR}, \mathrm{SD}$, or non-CR/non-PD (progressive disease). DCBC was achieved in 58 of 75 patients $(77.3 \%)$. Patients whose best overall response to this chemotherapy was not evaluable were treated as missing values in the following analysis related to DCBC.

Efficacy and safety of nivolumab. With regard to the best response to nivolumab, 1 patient achieved a CR (1.3\%), 7 $(8.8 \%)$ a $\mathrm{PR}$, and $18(22.5 \%)$ a $\mathrm{SD}$, with the remaining 54 patients experiencing PD (67.5\%), yielding an ORR and DCR of $10.0 \%$ (8 of 80 patients, $95 \%$ CI of $4.4-18.8 \%$ ) and $32.5 \%$ (26 of 80 patients, 95\% CI of 22.4-43.9\%), respectively. With a median follow-up time of 5.1 months, the median PFS was 1.63 months (95\% CI=1.28-1.84 months) and median OS was 5.30 months $(95 \% \mathrm{CI}=4.11$ 7.30 months) for the total study population. Immune-related adverse events of grade 3 or higher were observed in 1 patient, who developed both grade 3 colitis and grade 3 hypertransaminasemia; this was the only patient for whom nivolumab was discontinued as a result of adverse events.

Treatment after nivolumab. At the time of data cutoff, 73 $(91.3 \%)$ of the 80 patients had experienced PD on nivolumab, and $27(37.0 \%)$ of these 73 patients had received at least one subsequent therapy. The most common subsequent therapy was irinotecan monotherapy (13 of 27 patients, $48.1 \%$ ), followed by irinotecan plus ramucirumab ( 3 of 27 patients, 11.1\%), capecitabine plus oxaliplatin (3 of 27 patients, $11.1 \%$ ), and others ( 8 of 27 patients, $29.6 \%$ ).

Factors affecting nivolumab efficacy. Univariable Cox proportional hazards regression revealed that a GPS of 1 or 2 was significantly associated with a shorter PFS for nivolumab, and that DCBC was significantly associated with a longer PFS (Table II). An ECOG PS of 2, number of metastatic sites of $\geq 2$, the presence of liver metastasis, a high NLR, and a GPS of 1 or 2 were significantly associated with a shorter OS, whereas DCBC was significantly associated with a longer OS (Table II). Multivariable analysis (Table III) revealed that a GPS of 2 was associated with a shorter PFS $(\mathrm{HR}=1.91$ with a 95\% $\mathrm{CI}=0.94-3.89, p=0.074)$ and $\mathrm{OS}$ $(\mathrm{HR}=5.89$ with a $95 \% \mathrm{CI}=2.52-13.80, p<0.001)$, although only the latter association was significant. Conversely, DCBC was associated with a longer PFS (HR=0.51 with a $95 \% \mathrm{CI}=0.27-0.96, p=0.038)$ and $\mathrm{OS}(\mathrm{HR}=0.60$ with a $95 \%$ $\mathrm{CI}=0.31-1.16, p=0.125)$, with only the former association being significant.

Kaplan-Meier survival curves were clearly separated according to GPS, with a higher GPS being significantly associated with a shorter median PFS (3.0 vs. 1.6 vs. 1.4 
Table II. Univariable analysis of PFS and OS for nivolumab.

\begin{tabular}{|c|c|c|c|c|c|c|}
\hline \multirow[t]{2}{*}{ Characteristic } & \multicolumn{3}{|c|}{ PFS } & \multicolumn{3}{|c|}{ OS } \\
\hline & HR & $95 \% \mathrm{CI}$ & $p$-Value & HR & $95 \% \mathrm{CI}$ & $p$-Value \\
\hline Age, $\geq 75$ years $(v s .<75)$ & 0.84 & $0.51-1.38$ & 0.491 & 1.14 & $0.67-1.94$ & 0.625 \\
\hline Gender, female ( $v s$. male) & 0.89 & $0.47-1.69$ & 0.719 & 0.95 & $0.47-1.94$ & 0.893 \\
\hline ECOG PS, $2(v s .0$ or 1$)$ & 1.36 & $0.71-2.59$ & 0.358 & 2.22 & $1.14-4.32$ & 0.019 \\
\hline Histology, undifferentiated ( $v s$. differentiated) & 1.02 & $0.60-1.73$ & 0.944 & 1.21 & $0.69-2.13$ & 0.500 \\
\hline HER2 status, positive ( $v s$. negative) & 1.22 & $0.69-2.16$ & 0.501 & 0.80 & $0.41-1.59$ & 0.528 \\
\hline No. of metastatic sites, $\geq 2(v s .1)$ & 1.46 & $0.88-2.41$ & 0.142 & 1.78 & $1.02-3.10$ & 0.043 \\
\hline Liver metastasis, yes (vs. no) & 1.26 & $0.79-2.01$ & 0.336 & 1.73 & $1.03-2.91$ & 0.038 \\
\hline Previous gastrectomy, yes (vs. no) & 0.83 & $0.52-1.32$ & 0.426 & 0.99 & $0.60-1.64$ & 0.973 \\
\hline No. of previous regimens, $\geq 3$ ( $v s .2$ ) & 1.12 & $0.70-1.80$ & 0.631 & 1.24 & $0.74-2.08$ & 0.423 \\
\hline LDH level, $\geq$ median $(v s .<$ median $)$ & 1.39 & $0.87-2.20$ & 0.166 & 1.19 & $0.72-1.97$ & 0.487 \\
\hline Neutrophil/lymphocyte ratio, $\geq 5$ ( $v s .<5)$ & 1.70 & $0.98-2.95$ & 0.057 & 2.33 & $1.30-4.16$ & 0.004 \\
\hline \multicolumn{7}{|l|}{ GPS } \\
\hline $1(v s .0)$ & 1.78 & $1.04-3.04$ & 0.035 & 2.78 & $1.46-5.31$ & 0.002 \\
\hline $2(v s .0)$ & 2.67 & $1.43-4.97$ & 0.002 & 7.95 & $3.78-16.73$ & $<0.001$ \\
\hline DCBC, yes (vs. no) & 0.44 & $0.25-0.79$ & 0.006 & 0.43 & $0.23-0.78$ & 0.006 \\
\hline
\end{tabular}

PFS, Progression-free survival; OS, overall survival; HR, hazard ratio; CI, confidence interval; ECOG PS, Eastern Cooperative Oncology Group performance status; HER2, human epidermal growth factor receptor 2; LDH, lactate dehydrogenase; GPS, Glasgow prognostic score; DCBC, disease control by chemotherapy prior to nivolumab.

Table III. Multivariable analysis of PFS and OS for nivolumab.

\begin{tabular}{|c|c|c|c|c|c|c|}
\hline \multirow[t]{2}{*}{ Characteristic } & \multicolumn{3}{|c|}{ PFS } & \multicolumn{3}{|c|}{ OS } \\
\hline & HR & $95 \% \mathrm{CI}$ & $p$-Value & HR & $95 \% \mathrm{CI}$ & $p$-Value \\
\hline ECOG PS, 2 (vs. 0 or 1$)$ & & & & 2.09 & $0.91-4.80$ & 0.084 \\
\hline No. of metastatic sites, $\geq 2$ ( $v s .1)$ & 1.39 & $0.81-2.37$ & 0.229 & 1.84 & $0.90-3.78$ & 0.095 \\
\hline Liver metastasis, yes (vs. no) & & & & 1.15 & $0.58-2.28$ & 0.693 \\
\hline LDH level, $\geq$ median (vs. <median) & 1.41 & $0.87-2.30$ & 0.163 & & & \\
\hline Neutrophil/lymphocyte ratio, $\geq 5$ (vs. <5) & 1.61 & $0.87-2.98$ & 0.129 & 1.57 & $0.76-3.23$ & 0.219 \\
\hline \multicolumn{7}{|l|}{ GPS } \\
\hline $1(v s .0)$ & 1.49 & $0.83-2.69$ & 0.182 & 1.94 & $0.95-3.99$ & 0.070 \\
\hline $2(v s .0)$ & 1.91 & $0.94-3.89$ & 0.074 & 5.89 & $2.52-13.80$ & $<0.001$ \\
\hline DCBC, yes (vs. no) & 0.51 & $0.27-0.96$ & 0.038 & 0.60 & $0.31-1.16$ & 0.125 \\
\hline
\end{tabular}

PFS, Progression-free survival; OS, overall survival; HR, hazard ratio; CI, confidence interval; ECOG PS, Eastern Cooperative Oncology Group performance status; LDH, lactate dehydrogenase; GPS, Glasgow prognostic score; DCBC, disease control by chemotherapy prior to nivolumab.

months for a GPS of 0 vs. 1 vs. 2, respectively, $p=0.005)$ (Figure 2A) and OS (11.0 vs. 5.1 vs. 2.9 months for a GPS of $0 v s .1$ vs. 2, respectively, $p<0.001$ ) (Figure 2B). Such curves also showed a separation according to DCBC status, with patients who achieved DCBC having a significantly longer median PFS (1.7 vs. 1.4 months, $p=0.004)$ (Figure 2C) and OS (7.0 vs. 4.8 months, $p=0.005$ ) (Figure 2D). No significant difference in PFS or OS for nivolumab was apparent according to the type of chemotherapy regimen administered immediately prior to nivolumab treatment in patients who achieved DCBC (Figure 3).
Analysis of the association between the best OS to nivolumab and either GPS or DCBC status revealed that GPS was significantly associated with ORR (23.3\% vs. $3.1 \%$ vs. $0 \%$ for a GPS of $0 v s .1 v s .2$, respectively, $p=0.010$ ) and DCR (50.0\% vs. $25.0 \%$ vs. $16.7 \%$ for a GPS of 0 vs. 1 vs. 2 , respectively, $p=0.034)$, whereas DCBC was associated with ORR $(12.1 \%$ vs. $0 \%$ for $\mathrm{DCBC}+v s$. DCBC-, respectively, $p=0.339)$ and DCR (41.4\% vs. $5.9 \%$ for DCBC+ vs. DCBC-, respectively, $p=0.007)$, with only the latter association being significant.

Finally, we analyzed PFS and OS for nivolumab stratified according to both GPS and DCBC status. We found that a 

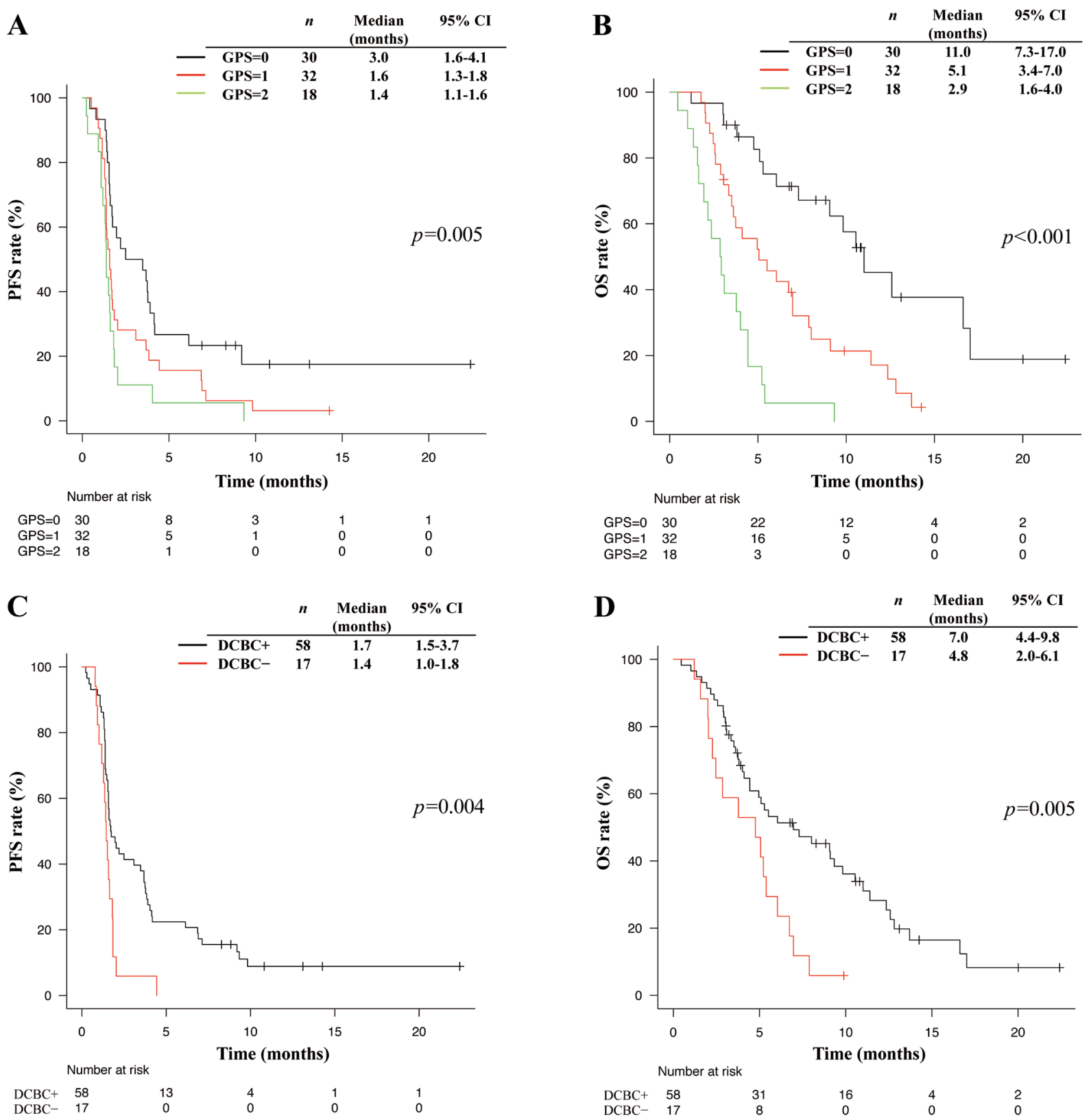

Figure 2. Kaplan-Meier analysis for progression-free survival (A), and overall survival (B) according to GPS. Kaplan-Meier analysis of progressionfree survival $(C)$ and overall survival $(D)$ according to whether $D C B C$ was achieved $(D C B C+)$ or not $(D C B C-)$. $p$-Values were calculated with the log-rank test. GPS, Glasgow prognostic score; DCBC, disease control by the chemotherapy just prior nivolumab; PFS, progression-free survival; OS, overall survival; CI, confidence interval.

higher GPS tended to be associated with a shorter median PFS (3.7 vs. $1.6 v s .1 .3$ months for a GPS of $0 v s .1$ vs. 2 , respectively) and OS (11.0 vs. 5.5 vs. 2.4 months for a GPS of 0 vs. 1 vs. 2, respectively) in patients who achieved $\mathrm{DCBC}$, whereas such a trend was not apparent for patients who did not achieve DCBC (Figure 4).

\section{Discussion}

On the basis of the results of the pivotal ATTRACTION-2 trial (5), that established nivolumab as standard therapy for heavily pretreated patients with AGC, nivolumab monotherapy is now administered in Japan as a third- or 


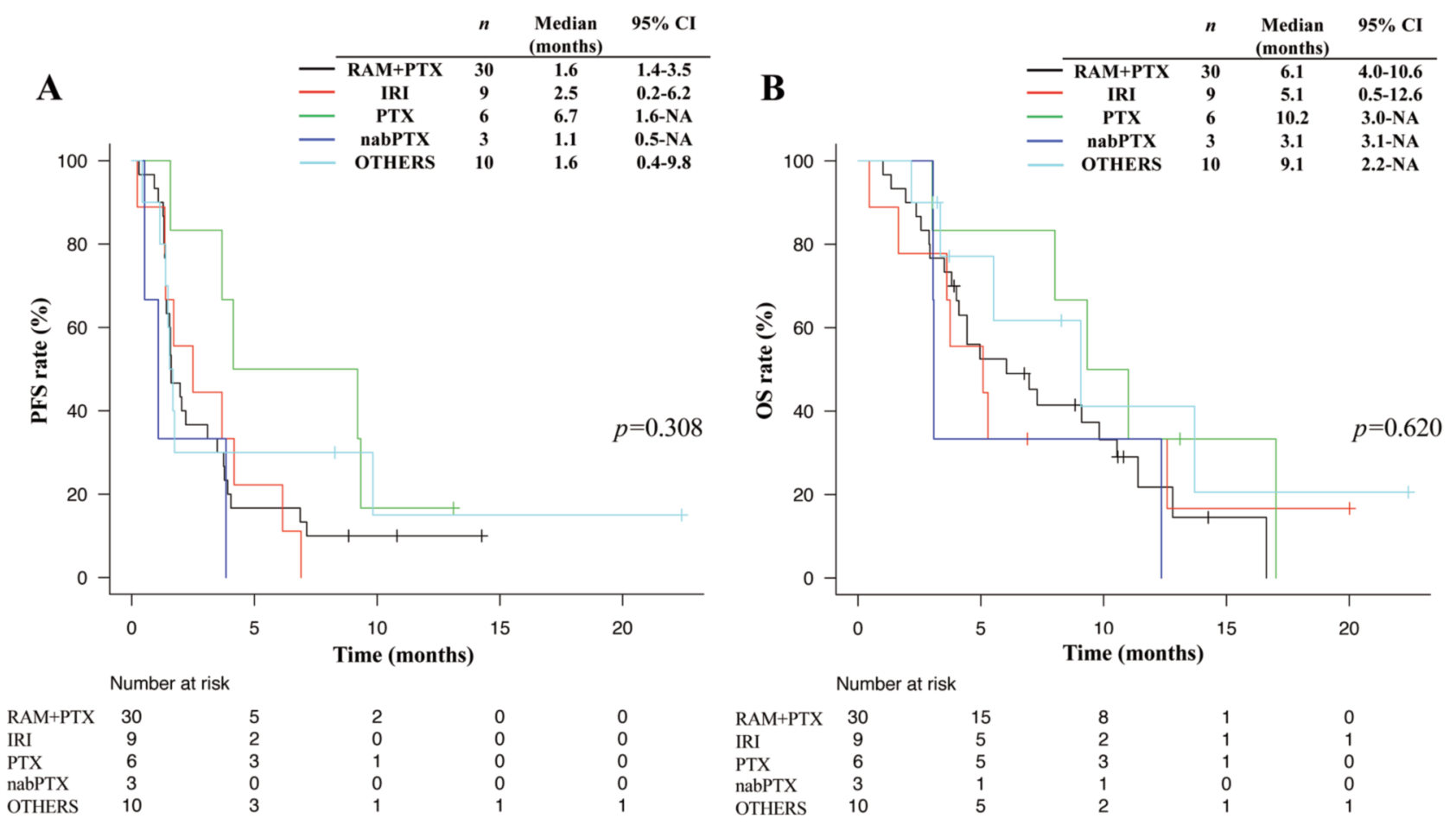

Figure 3. Kaplan-Meier analysis for progression-free survival $(A)$ and overall survival $(B)$ according to the chemotherapy regimen just prior nivolumab in patients who achieved disease control by the chemotherapy just prior nivolumab. p-Values were calculated with the log-rank test. $R A M+P T X$, ramucirumab plus paclitaxel; IRI, irinotecan monotherapy; PTX, paclitaxel monotherapy; nabPTX, nanoparticle albumin-bound paclitaxel monotherapy; PFS, progression free survival; OS, overall survival; CI, confidence interval.

later-line treatment for AGC. The efficacy of nivolumab monotherapy for our study population in the clinical practice setting was essentially consistent with that reported in ATTRACTION-2. We now identified GPS and DCBC as effective biomarkers of nivolumab monotherapy for AGC.

GPS has been widely adopted as a systemic inflammatory and nutritional marker (10), with the score before treatment having been shown to be a prognostic factor not only for postoperative survival in gastric cancer patients but also for survival in such patients receiving palliative chemotherapy (19). Given that recent studies have suggested that cancerrelated inflammation suppresses effective antitumor immunity through multiple pathways (20), GPS may serve as a biomarker to reflect sensitivity to immunotherapy. However, to the best of our knowledge, our study is the first to show a significant association between GPS and nivolumab efficacy for AGC. Our multivariable analysis revealed that a higher GPS was significantly associated with a shorter OS and tended to be associated with a shorter PFS. Moreover, a higher GPS was significantly associated with a lower ORR and DCR. Together, these findings suggest that GPS may be a predictive factor as well as a prognostic factor for nivolumab monotherapy in AGC, and this potential of GPS thus warrants further study.
We also found that DCBC was associated with the efficacy of nivolumab for AGC, consistent with previous findings in non-small cell lung cancer (16). Multivariable analysis revealed that patients who achieved DCBC had a significantly longer PFS and tended to have a longer OS for nivolumab compared to those who did not. DCBC was also associated with a higher ORR and significantly associated with a higher DCR for nivolumab, suggesting that chemotherapy immediately prior to nivolumab treatment can have an immunostimulatory effect and thereby enhance the response to nivolumab. Paclitaxel and irinotecan were the two most commonly administered chemotherapeutic agents just before nivolumab treatment, with these two drugs having been found to enhance the antitumor immune response through different pathways (21-24). Further studies are warranted to identify chemotherapeutic regimens with the largest effects on the efficacy of subsequent nivolumab therapy, although no significant differences among regimens in this regard were apparent in the present study.

We found that GPS was associated with PFS and OS for nivolumab in patients who achieved DCBC, but not in those who did not. Our data thus suggest the importance of changes 


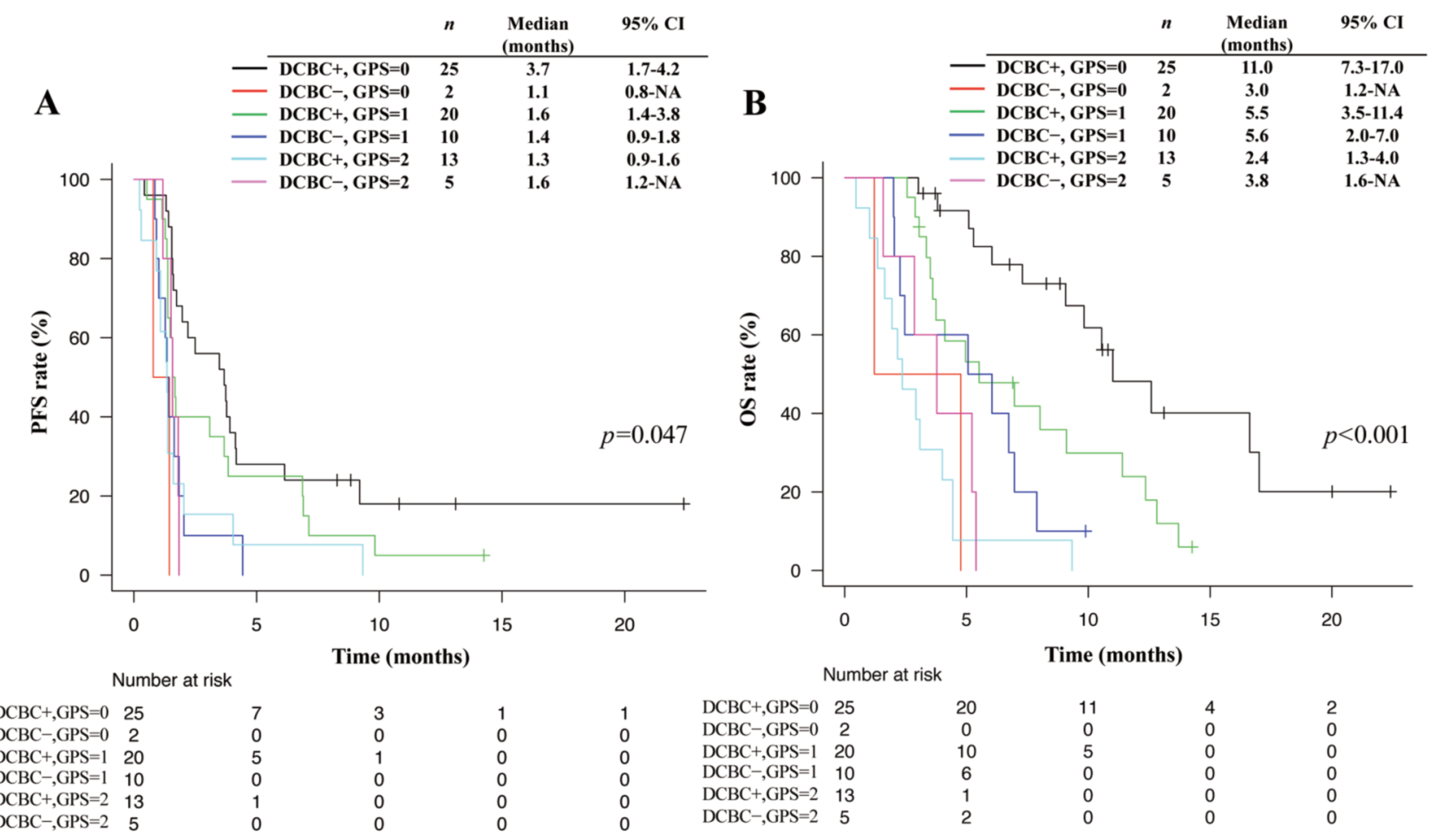

Figure 4. Kaplan-Meier analysis for progression-free survival (A) and overall survival (B) according to GPS stratified by DCBC+/-.p-Values were calculated with the log-rank test. GPS, Glasgow prognostic score; DCBC, disease control by the chemotherapy just prior to nivolumab; PFS, progression-free survival; OS, overall survival; CI, confidence interval.

in serum albumin and CRP levels during chemotherapy as a determinant of when to switch to nivolumab monotherapy in patients who have achieved disease control, especially in those without measurable lesions.

Both of GPS and DCBC have several advantages relevant to clinical practice. First, they are readily accessible and thus potentially widely applicable. Second, although examinations for PD-L1 expression, MSI or Epstein-Barr virus positivity require technical expertise, GPS and DCBC do not. Finally, with the use of GPS and DCBC, prediction can be made prior to administration of nivolumab, unlike previously suggested biomarkers such as development of immune-related adverse events (25). On the other hand, the following disadvantages should be taken into account: GPS is intrinsically susceptible to transient inflammation including acute infection, and DCBC can be utilized only when the information of response to chemotherapy immediately prior to nivolumab is available.

Our study has several limitations. First, it was retrospective in nature and lacked a control group of patients who did not receive nivolumab. The identified biomarkers (GPS and DCBC) may thus not necessarily be specific to nivolumab. Second, we did not assess molecular biomarkers such as MSI-H and PD-L1 expression and so did not take these factors into account in our analysis. However, evidence suggests that not a few AGC patients with stable microsatellite or PD-L1-lacking tumors indeed respond to ICIs $(5,7)$. Third, we investigated the efficacy of only nivolumab, and it remains to be determined whether GPS and DCBC might also be related to immunotherapies other than nivolumab monotherapy. Given that a recent study demonstrated a noninferior survival benefit for the ICI pembrolizumab compared to chemotherapy in the first-line setting for AGC (26), our data suggest that GPS might also identify patients likely to benefit from pembrolizumab monotherapy in this setting. Moreover, several phase III studies to evaluate the efficacy of ICIs plus chemotherapy in the first-line setting are ongoing. Further studies on the relation of GPS to ICI efficacy are therefore warranted.

In conclusion, we identified GPS and DCBC as potential biomarkers of nivolumab monotherapy in the third or laterline setting for AGC. Further study is warranted to determine whether GPS might be suitable as a predictive or prognostic biomarker of immunotherapies in earlier-line settings or in combination with chemotherapeutic agents. 


\section{Conflicts of Interest}

H.K. has received consulting fees from Bristol-Myers Squibb Co. Ltd., Eli Lilly Japan K.K., MSD K.K., Ono Pharmaceutical Co. Ltd., Daiichi-Sankyo Co. Ltd., and Taiho Pharmaceutical Co. Ltd.; honoraria from Bristol-Myers Squibb Co. Ltd., AstraZeneca K.K., Bayer yakuhin Ltd., Eli Lilly Japan K.K., MSD K.K., Ono Pharmaceutical Co. Ltd., Chugai Pharmaceutical Co. Ltd., Daiichi Sankyo Co. Ltd., Takeda Pharmaceutical Co. Ltd., and Taiho Pharmaceutical Co. Ltd.; lecture fees from Bristol-Myers Squibb Co. Ltd., Eli Lilly Japan K.K., MSD K.K., Ono Pharmaceutical Co. Ltd., Chugai Pharmaceutical Co. Ltd., Takeda Pharmaceutical Co. Ltd., and Taiho Pharmaceutical Co. Ltd.; and research funding from Chugai Pharmaceutical Co. Ltd. and Eisai Co. Ltd. S.M. has received personal fees from Taiho Pharmaceutical Co., Eli Lilly, Takeda Pharmaceutical Co. and Ono Pharmaceutical Co. T.Tamura has received honoraria from Merck Serono Co. Ltd., speaker's bureau fees from Daiichi Sankyo Co. Ltd., and research funding from Takeda Pharmaceutical Co. Ltd., Chugai Pharmaceutical Co. Ltd., and Taiho Pharmaceutical Co. Ltd. K.N. has received honoraria from Astellas Pharma Inc., Takeda Pharmaceutical Co. Ltd., AstraZeneca K.K., Chugai Pharmaceutical Co. Ltd., MSD K.K., Eli Lilly Japan K.K., Ono Pharmaceutical Co. Ltd., Nippon Boehringer Ingelheim Co. Ltd., Daiichi Sankyo Co. Ltd., Novartis Pharma K.K., Taiho Pharmaceutical Co. Ltd., Pfizer Japan Inc., Bristol Myers Squibb Company, Nikkei Business Publications, Inc., KYORIN Pharmaceutical Co. Ltd., Thermo Fisher Scientific K.K., CareNet, Inc, NANZANDO Co. Ltd, Nichi-Iko Pharmaceutical Co. Ltd., Medical Review Co. Ltd., Hisamitsu Pharmaceutical Co. Inc., YOMIURI TELECASTING CORPORATION., YODOSHA CO. LTD., Reno. Medical K.K., Clinical Trial Co. Ltd., SymBio Pharmaceuticals Limited., MEDICUS SHUPPAN, Publishers Co. Ltd., AYUMI Pharmaceutical Corporation, Clinical Trial Co. Ltd.; and research funding from MSD K.K., AstraZeneca K.K., ICON Japan K.K., Astellas Pharma Inc., Takeda Pharmaceutical Co. Ltd., Novartis Pharma K.K., Eli Lilly Japan K.K., Quintiles Inc., Bristol Myers Squibb Company, CMIC Shift Zero K.K., Taiho Pharmaceutical Co. Ltd., Eisai Co. Ltd., PAREXEL International Corp., Nippon Boehringer Ingelheim Co. Ltd., Ono Pharmaceutical Co. Ltd., Kissei Pharmaceutical Co. Ltd., IQVIA Services JAPAN K.K., Pfizer Japan Inc., A2 Healthcare Corp., Kyowa Hakko Kirin Co. Ltd., AbbVie Inc., EPS Corporation., Chugai Pharmaceutical Co. Ltd., Daiichi Sankyo Co. Ltd., SymBio Pharmaceuticals Limited., Bayer Yakuhin, Ltd., Merck Serono Co. Ltd., inVentiv Health Japan, EPCRSU CO. LTD., GRITSONE ONCOLOGY.INC, Linical Co. Ltd., GlaxoSmithKline K.K., Otsuka Pharmaceutical Co. Ltd., Yakult Honsha Co. Ltd., EPS International Co. Ltd., Covance Inc. The other authors declare no conflict of interest.

\section{Authors' Contributions}

T.K., K.H., and S.M. conceptualized and designed the study. T.K., K.H., S.M., R.K., T Takahama, Y.N., S.F., T.O., and T Tamura involved in acquisition of data. T.K., K.H., S.M., Y.C., and K.N. involved in analysis and interpretation of data. All the authors were involved in manuscript for all aspects of the work.

\section{Acknowledgements}

The Authors sincerely appreciate all the patients who received treatment and were enrolled in this study.

\section{References}

1 Borghaei H, Paz-Ares L, Horn L, Spigel DR, Steins M, Ready NE, Chow LQ, Vokes EE, Felip E, Holgado E, Barlesi F, Kohlhaufl M, Arrieta O, Burgio MA, Fayette J, Lena H, Poddubskaya E, Gerber DE, Gettinger SN, Rudin CM, Rizvi N, Crino L, Blumenschein GR, Jr., Antonia SJ, Dorange C, Harbison CT, Graf Finckenstein F and Brahmer JR: Nivolumab $v s$. docetaxel in advanced nonsquamous non-small-cell lung cancer. N Engl J Med 373(17): 1627-1639, 2015. PMID: 26412456. DOI: 10.1056/NEJMoa1507643

2 Robert C, Long GV, Brady B, Dutriaux C, Maio M, Mortier L, Hassel JC, Rutkowski P, McNeil C, Kalinka-Warzocha E, Savage KJ, Hernberg MM, Lebbe C, Charles J, Mihalcioiu C, Chiarion-Sileni V, Mauch C, Cognetti F, Arance A, Schmidt H, Schadendorf D, Gogas H, Lundgren-Eriksson L, Horak C, Sharkey B, Waxman IM, Atkinson V and Ascierto PA: Nivolumab in previously untreated melanoma without BRAF mutation. N Engl J Med 372(4): 320-330, 2015. PMID: 25399552. DOI: $10.1056 /$ NEJMoa1412082

3 Motzer RJ, Escudier B, McDermott DF, George S, Hammers HJ, Srinivas S, Tykodi SS, Sosman JA, Procopio G, Plimack ER, Castellano D, Choueiri TK, Gurney H, Donskov F, Bono P, Wagstaff J, Gauler TC, Ueda T, Tomita Y, Schutz FA, Kollmannsberger C, Larkin J, Ravaud A, Simon JS, Xu LA, Waxman IM and Sharma P: Nivolumab vs. everolimus in advanced renal-cell carcinoma. N Engl J Med 373(19): $1803-$ 1813, 2015. PMID: 26406148. DOI: 10.1056/NEJMoa1510665

4 Schmid P, Adams S, Rugo HS, Schneeweiss A, Barrios CH, Iwata H, Dieras V, Hegg R, Im SA, Shaw Wright G, Henschel V, Molinero L, Chui SY, Funke R, Husain A, Winer EP, Loi S and Emens LA: Atezolizumab and nab-paclitaxel in advanced triple-negative breast cancer. N Engl J Med 379(22): 2108-2121, 2018. PMID: 30345906. DOI: 10.1056/NEJMoa1809615

5 Kang YK, Boku N, Satoh T, Ryu MH, Chao Y, Kato K, Chung HC, Chen JS, Muro K, Kang WK, Yeh KH, Yoshikawa T, Oh SC, Bai LY, Tamura T, Lee KW, Hamamoto Y, Kim JG, Chin K, Oh DY, Minashi K, Cho JY, Tsuda M and Chen LT: Nivolumab in patients with advanced gastric or gastro-oesophageal junction cancer refractory to, or intolerant of, at least two previous chemotherapy regimens (ONO-4538-12, ATTRACTION-2): A randomised, double-blind, placebo-controlled, phase 3 trial. Lancet 390(10111): 2461-2471, 2017. PMID: 28993052. DOI: 10.1016/S0140-6736(17)31827-5

6 Marabelle A, Le DT, Ascierto PA, Di Giacomo AM, De JesusAcosta A, Delord JP, Geva R, Gottfried M, Penel N, Hansen AR, Piha-Paul SA, Doi T, Gao B, Chung HC, Lopez-Martin J, Bang YJ, Frommer RS, Shah M, Ghori R, Joe AK, Pruitt SK and Diaz LA, Jr.: Efficacy of pembrolizumab in patients with noncolorectal high microsatellite instability/mismatch repairdeficient cancer: Results from the phase II KEYNOTE-158 study. J Clin Oncol 38(1): 1-10, 2020. PMID: 31682550. DOI: 10.1200/jco.19.02105

7 Shitara K, Ozguroglu M, Bang YJ, Di Bartolomeo M, Mandala M, Ryu MH, Fornaro L, Olesinski T, Caglevic C, Chung HC, Muro K, Goekkurt E, Mansoor W, McDermott RS, ShachamShmueli E, Chen X, Mayo C, Kang SP, Ohtsu A and Fuchs CS: Pembrolizumab vs. paclitaxel for previously treated, advanced gastric or gastro-oesophageal junction cancer (KEYNOTE-061): A randomised, open-label, controlled, phase 3 trial. Lancet 
392(10142): 123-133, 2018. PMID: 29880231. DOI: 10.1016/ S0140-6736(18)31257-1

8 Kim ST, Cristescu R, Bass AJ, Kim KM, Odegaard JI, Kim K, Liu XQ, Sher X, Jung H, Lee M, Lee S, Park SH, Park JO, Park YS, Lim HY, Lee H, Choi M, Talasaz A, Kang PS, Cheng J, Loboda A, Lee J and Kang WK: Comprehensive molecular characterization of clinical responses to PD-1 inhibition in metastatic gastric cancer. Nat Med 24(9): 1449-1458, 2018. PMID: 30013197. DOI: 10.1038/s41591-018-0101-z

9 Mishima S, Kawazoe A, Nakamura Y, Sasaki A, Kotani D, Kuboki Y, Bando H, Kojima T, Doi T, Ohtsu A, Yoshino T, Kuwata T, Tsuji A and Shitara K: Clinicopathological and molecular features of responders to nivolumab for patients with advanced gastric cancer. J Immunother Cancer 7(1): 24, 2019. PMID: 30704511. DOI: 10.1186/s40425-019-0514-3

10 Forrest LM, McMillan DC, McArdle CS, Angerson WJ and Dunlop DJ: Evaluation of cumulative prognostic scores based on the systemic inflammatory response in patients with inoperable non-small-cell lung cancer. Br J Cancer 89(6): 10281030, 2003. PMID: 12966420. DOI: 10.1038/sj.bjc.6601242

11 Crumley AB, McMillan DC, McKernan M, McDonald AC and Stuart RC: Evaluation of an inflammation-based prognostic score in patients with inoperable gastro-oesophageal cancer. $\mathrm{Br}$ J Cancer 94(5): 637-641, 2006. PMID: 16479253. DOI: $10.1038 /$ sj.bjc. 6602998

12 Glen P, Jamieson NB, McMillan DC, Carter R, Imrie CW and McKay CJ: Evaluation of an inflammation-based prognostic score in patients with inoperable pancreatic cancer. Pancreatology 6(5): 450-453, 2006. PMID: 16847382. DOI: $10.1159 / 000094562$

13 McMillan DC, Crozier JE, Canna K, Angerson WJ and McArdle CS: Evaluation of an inflammation-based prognostic score (GPS) in patients undergoing resection for colon and rectal cancer. Int J Colorectal Dis 22(8): 881-886, 2007. PMID: 17245566. DOI: $10.1007 / \mathrm{s} 00384-006-0259-6$

14 Wang D-s, Ren C, Qiu M-z, Luo H-y, Wang Z-q, Zhang D-s, Wang F-h, Li Y-h and Xu R-h: Comparison of the prognostic value of various preoperative inflammation-based factors in patients with stage III gastric cancer. Tumour Biol 33(3): 749756, 2012. PMID: 22198641. DOI: 10.1007/s13277-011-0285-z

15 Voorwerk L, Slagter M, Horlings HM, Sikorska K, van de Vijver KK, de Maaker M, Nederlof I, Kluin RJC, Warren S, Ong S, Wiersma TG, Russell NS, Lalezari F, Schouten PC, Bakker NAM, Ketelaars SLC, Peters D, Lange CAH, van Werkhoven E, van Tinteren H, Mandjes IAM, Kemper I, Onderwater S, Chalabi M, Wilgenhof S, Haanen J, Salgado R, de Visser KE, Sonke GS, Wessels LFA, Linn SC, Schumacher TN, Blank CU and Kok M: Immune induction strategies in metastatic triple-negative breast cancer to enhance the sensitivity to PD-1 blockade: The tonic trial. Nat Med 25(6): 920-928, 2019. PMID: 31086347. DOI: 10.1038/s41591-019-0432-4

16 Nakahama K, Isa S, Tamiya A, Taniguchi Y, Shiroyama T, Suzuki H, Inoue T, Tamiya M, Hirashima T, Imamura F and Atagi S: The association between chemotherapy immediately before nivolumab and outcomes thereafter. Anticancer Res 37(10): 5885-5891, 2017. PMID: 28982916. DOI: 10.21873/ anticanres. 12034

17 Ogata T, Satake H, Ogata M, Hatachi Y, Inoue K, Hamada M and Yasui H: Neutrophil-to-lymphocyte ratio as a predictive or prognostic factor for gastric cancer treated with nivolumab: A multicenter retrospective study. Oncotarget 9(77): 34520-34527, 2018. PMID: 30349646. DOI: 10.18632/oncotarget.26145

18 Sacdalan DB, Lucero JA and Sacdalan DL: Prognostic utility of baseline neutrophil-to-lymphocyte ratio in patients receiving immune checkpoint inhibitors: A review and meta-analysis. Onco Targets Ther 11: 955-965, 2018. PMID: 29503570. DOI: $10.2147 /$ ott.S153290

19 Zhang CX, Wang SY, Chen SQ, Yang SL, Wan L and Xiong B: Association between pretreatment glasgow prognostic score and gastric cancer survival and clinicopathological features: A metaanalysis. Onco Targets Ther 9: 3883-3891, 2016. PMID: 27390529. DOI: $10.2147 /$ ott.S103996

20 Mantovani A, Allavena P, Sica A and Balkwill F: Cancer-related inflammation. Nature 454(7203): 436-444, 2008. PMID: 18650914. DOI: $10.1038 /$ nature07205

21 Heinhuis KM, Ros W, Kok M, Steeghs N, Beijnen JH and Schellens JHM: Enhancing antitumor response by combining immune checkpoint inhibitors with chemotherapy in solid tumors. Ann Oncol 30(2): 219-235, 2019. PMID: 30608567. DOI: $10.1093 /$ annonc/mdy551

22 Zhang L, Dermawan K, Jin M, Liu R, Zheng H, Xu L, Zhang Y, Cai Y, Chu Y and Xiong S: Differential impairment of regulatory $\mathrm{T}$ cells rather than effector $\mathrm{T}$ cells by paclitaxel-based chemotherapy. Clin Immunol 129(2): 219-229, 2008. PMID: 18771959. DOI: $10.1016 /$ j.clim.2008.07.013

23 Demaria S, Volm MD, Shapiro RL, Yee HT, Oratz R, Formenti SC, Muggia F and Symmans WF: Development of tumorinfiltrating lymphocytes in breast cancer after neoadjuvant paclitaxel chemotherapy. Clin Cancer Res 7(10): 3025-3030, 2001. PMID: 11595690.

24 McKenzie JA, Mbofung RM, Malu S, Zhang M, Ashkin E, Devi S, Williams L, Tieu T, Peng W, Pradeep S, Xu C, Zorro Manrique S, Liu C, Huang L, Chen Y, Forget MA, Haymaker C, Bernatchez C, Satani N, Muller F, Roszik J, Kalra A, Heffernan T, Sood A, Hu J, Amaria R, Davis RE and Hwu P: The effect of topoisomerase I inhibitors on the efficacy of T-cell-based cancer immunotherapy. J Natl Cancer Inst 110(7): 777-786, 2018. PMID: 29267866. DOI: 10.1093/jnci/djx257

25 Masuda K, Shoji H, Nagashima K, Yamamoto S, Ishikawa M, Imazeki H, Aoki M, Miyamoto T, Hirano H, Honma Y, Iwasa S, Okita N, Takashima A, Kato K and Boku N: Correlation between immune-related adverse events and prognosis in patients with gastric cancer treated with nivolumab. BMC Cancer 19(1): 974, 2019. PMID: 31638948. DOI: 10.1186/s12885-019-6150-y

26 Tabernero J, Cutsem EV, Bang Y-J, Fuchs CS, Wyrwicz L, Lee KW, Kudaba I, Garrido M, Chung HC, Salguero HRC, Mansoor W, Braghiroli MIFM, Goekkurt E, Chao J, Wainberg ZA, Kher U, Shah S, Kang SP and Shitara K: Pembrolizumab with or without chemotherapy $v s$. chemotherapy for advanced gastric or gastroesophageal junction (G/GEJ) adenocarcinoma: The phase III keynote-062 study. J Clin Oncol 37(18_suppl): LBA4007LBA4007, 2019. DOI: 10.1200/JCO.2019.37.18_suppl.LBA4007

Received March 31, 2020

Revised April 11, 2020

Accepted April 16, 2020 